\title{
FLUCTUATIONS IN CONFIDENCE AND ASYMMETRIC BUSINESS CYCLES
}

\author{
SIMON M. POTTER ${ }^{1}$ \\ Domestic Research Function \\ Federal Reserve Bank of New York, \\ 33 Liberty St. \\ New York, NY 10045-0001, U.S.A. \\ E-mail: simon.potter@ny.frb.org \\ tel: $212-720-6309$ \\ fax: 212-720-1844
}

February 1999

\begin{abstract}
There is now a great deal of empirical evidence that business cycle fluctuations contain asymmetries. The asymmetries found in post-war US data are inconsistent with the behavior of the US economy in the Great Depression. In a model where business cycle asymmetries are produced by rational fluctuations in the confidence of investors, I examine whether this inconsistency can be explained by differences in government policy. It is found that the 'ineptness' of government intervention during the Great Depression in reducing the confidence of investors rather than the success of post-war stabilization policy in raising confidence is the most likely explanation.
\end{abstract}

JEL: E32, C22

keywords: asymmetry, confidence, business cycle, great depression

\footnotetext{
${ }^{1}$ Numerous discussions with Joe Ostroy have had a deep influence on this paper. The views expressed in this paper are those of the author and do not necessarily reflect the views of the Federal Reserve Bank of New York or the Federal Reserve System.
} 


\section{Introduction}

Early analysts of economic fluctuations such as Keynes, Mitchell, Burns and Pigou all discussed asymmetries over the business cycle. Perhaps the most well-known description of these asymmetries is by Keynes. ...There is, however, another characteristic of what we call the trade cycle which our explanation must cover; namely, the phenomenon of the crisis - the fact that the substitution of a downward for an upward tendency often takes place suddenly and violently, whereas there is as a rule, no such sharp turning points when an upward is substituted for a downward tendency. In this paper I examine asymmetries over the business cycle driven by fluctuations in the confidence of investors.

Despite the widespread belief in asymmetries amongst early business cycle analysts, little formal statistical evidence of the extent of the asymmetries was presented until Neftci's (1984) analysis of postwar US unemployment rates. Extending the approach of Neftci, Hamilton (1989) showed that the business cycle could be modeled as abrupt shifts between fast and slow growth rates. Other work, prompted by Hamilton's contribution, found strong evidence for an additional asymmetry in the propagation mechanism of post-second world war US output. Beaudry and Koop (1993), Tiao and Tsay (1994) and Potter (1995) all find evidence that negative shocks to output during recessions tend to have a negligible or even a positive effect on predictions of output at one year or longer horizons. This effect is illustrated in Figure 1 by the impulse response in the deepest recession in the post-second world war period, 1980 Quarter 2 and the model estimated by Potter. The impulse response to a shock of $-2 \%$ hitting during this recession period is positive after four quarters.

Few theoretical explanations of asymmetries over the business cycle have been offered. ${ }^{1}$ For most macroeconomists, asymmetric business cycles are a sideshow that do not contribute to the fundamental question of understanding the sources of business cycle fluctuations. In this light, consider the findings of negative shocks being less persistent in recessions than in expansions. This suggests a very different asymmetry in post-war data than the one that Keynes perceived in the pre-war data. Keynes was writing

\footnotetext{
${ }^{1}$ Many business cycle models of the 1950s did focus on nonlinearities associated with floors and ceilings on output growth that could produce such asymmetries. However, no tight microfoundations for the floors and ceilings were given. Chalkley and Lee (1998) develop a model of asymmetric business cycles based on learning and information aggregation.
} 


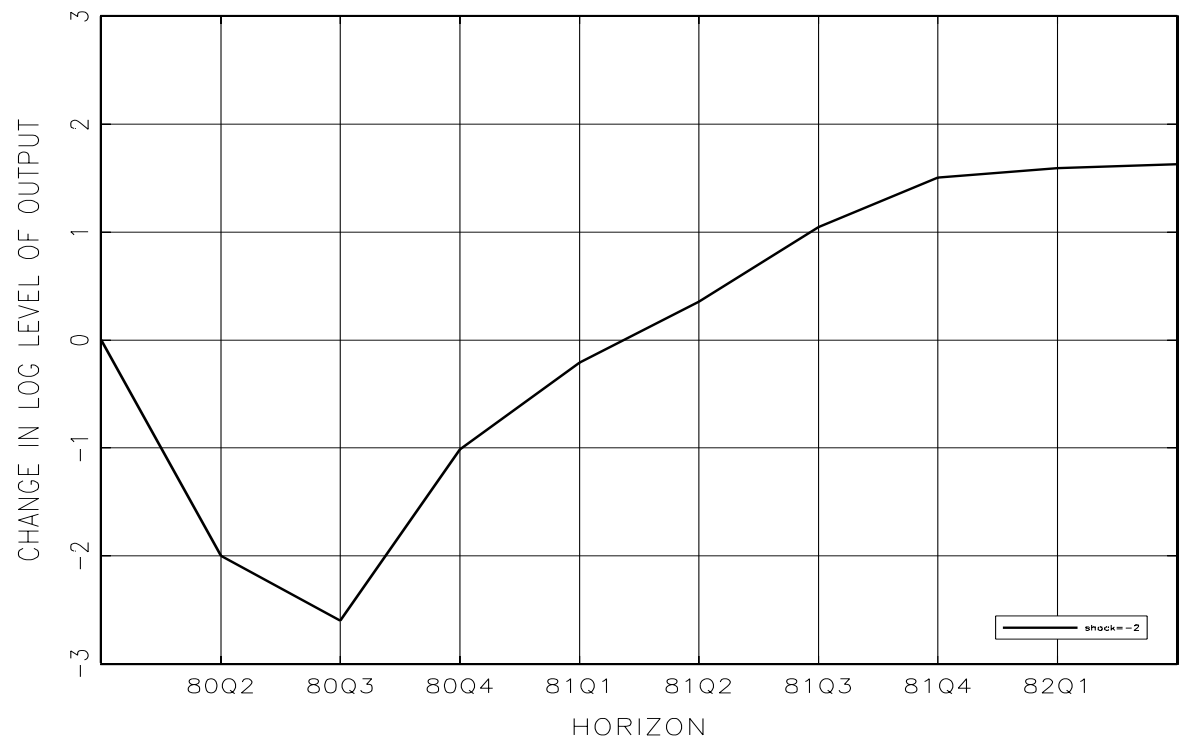

Figure 1: Nonlinear Impulse Response for 1980 Quarter Two

The impulse response is calculated as $E\left[Y_{t+n} \mid Y_{t}-2, Y_{t-1}, Y_{t-3}\right]-E\left[Y_{t+n} \mid Y_{t}, Y_{t-1}, Y_{t-3}\right]$, where $Y_{t}$ is the 100 times the logarithm of US GDP and $t=1980$ Quarter 2.

having observed the persistent slumps of the 1920s and 1930s, in particular the Great Depression in the United States.

Potter (1995) uses simulation to show that if the pre-war propagation mechanism had similar properties with respect to negative shocks as the post-war mechanism estimated by his model, then a contraction of Great Depression size would not have occurred. Figure 2 shows the result of repeating this simulation with the model estimated by Beaudry and Koop. Once again the conclusion is the same. Given we know that the Great Depression did occur, we have two types of business cycle asymmetries: In the pre-war period the reaction to large negative shocks was a prolonged slump in economy activity. In the post-war period, although we have not experienced negative shocks of Great Depression size, simulations show that the reaction to negative shocks during recession periods is not a prolonged slump in economic 
activity.

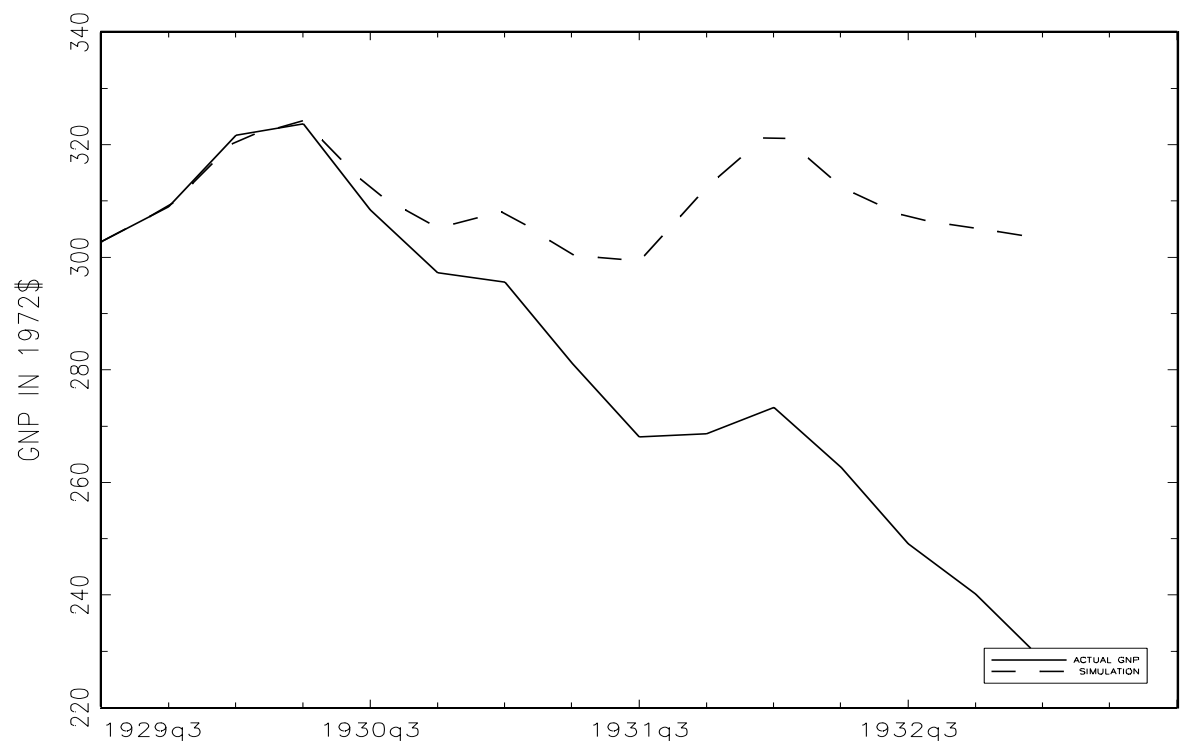

Figure 2: The Great Depression and a Simulation

The simulation is conducted by taking the parameter estimates of the propagation mechanism of Beaudry and Koop estimated on post-war time series and using impulses estimated from the Great Depression period from Potter (1995).

One explanation of these findings is the success of stabilization policy in the post-war period. Not only has stabilization policy reduced the size of negative shocks hitting the economy, but it has also has instituted automatic stabilizers that reverse the effect of negative shocks during recessions. An alternative explanation for the findings on asymmetries, as argued by Friedman and Schwartz (1963), is that the Great Depression was an extraordinary event partly caused by inept actions of the Federal Reserve Board. Further, once one removes the Great Depression from the sample, the pre-war period shows the same resistance to negative shocks as the post-war period. Friedman (1993) called this the plucking theory of 
business cycles: The deeper the recession, the faster the subsequent recovery. ${ }^{2}$

The corridor analogy of Leijonhufvud (1981) provides a useful means of examining these issues within a single class of models. ${ }^{3}$ Leijonhufvud's view was that the economy had a full coordination time path along which all agents correctly perceive the opportunities for social efficient trade. Shocks tend to displace the economy from this path and introduce deviations between the private perceptions of the gains to trade and the social perception based on complete information. Within a corridor of deviations from the full coordination path the displacement is corrected by market forces, with the force of the correction increasing with the distance from the full coordination path. However, if the displacement from the full coordination path is sufficiently large, the coordination problems produced by the divergence between the private perceptions of the gains from trade and the social perception based on complete information become so wide that market forces are unable to return the economy to the full coordination path.

For an economy to have the corridor property, two features are essential. First, there needs to be an externality that produces differences between private perceptions of the cost/benefits of various trades and social cost/benefits. Second, the importance of the externality must vary over the business cycle. Obviously it makes sense that during recessions the externality is more important than in expansions. But, in addition, a more subtle property is required. For recessions within the corridor there must be forces in operation that counteract the externality. For recessions outside the corridor these self-correcting forces need to fail. ${ }^{4}$

Along the lines of Leijonhufvud's views, I consider an externality produced by the failure of agents to

\footnotetext{
${ }^{2}$ Balke and Wynne (1994) find statistical evidence in favor of the plucking model going back to the nineteenth century.

${ }^{3}$ One could capture some of the aspects of the plucking model of business cycles using the dynamics of the standard neoclassical growth model. As the economy is shocked below its steady state mean growth path, the marginal product of capital starts to rise. Thus, when good productivity shock occurs the economy experiences faster than average growth as it returns to the steady state path. However, the standard one sector model is not be capable of producing the dynamics shown in Figure 1 where in a recession, a negative shock leads to predictions of higher growth than if the shock had not occurred. Further, the one sector model offers no obvious explanation of the Great Depression.

${ }^{4}$ In the recent literature on coordination failure a number of possible externalities have been suggested (see Cooper and John, 1988) for an overview. For example, the social level of knowledge might enter into the production functions of individual firms. A difficulty with many of these models of coordination failure is the movement between equilibria of low levels of coordination failure and equilibrium of full coordination is not explicitly modeled. Rather an emphasis has been placed on self-fulfilling expectations triggered by exogenous sunspots.
} 
take into account that their actions produce valuable information. ${ }^{5}$ The action for which the information is required will be an irreversible investment of resources into a production technique. Individual production techniques per se are neither good nor bad. Instead their value depends on an unobserved factor. With the appropriate choice of production technique to match the unobserved factor, productivity will be high on average. With inappropriate choices, productivity will be low on average. Thus, by observing the productivity of investment over a sufficiently long period of time, the value of the unobserved factor associated with investment will be revealed.

Agents form rational beliefs using Bayes' rule about the unobserved factor associated with their investment opportunity. The unobserved factor varies across individual investment opportunities according to a two state Markov process. Individual investors receive private signals about the efficiency of their investment opportunities. Observation of previous investment choices and subsequent output allow investors to form improved estimates of the current state of the unobserved factor. I show that this information aggregation problem rather than being undertaken by individual agents can be achieved by the stock market. That is, individual investors can base their investment decisions on the recent behavior of the stock market.

The externality is produced by alternative production techniques generating differing amounts of information to future investors. Some of the techniques produce a great deal of information about the unobserved factor, others produce little information. One type of production technique is available that does not depend on the unobservable factor. Hence, it produces no information for other agents. In addition it is assumed that agents invest only once and in a sequential order. Further, I assume that there is no merging or acquisition of future investments available on the stock market. Thus, individual agents are not allowed to internalize the externality.

In order to get the strength of the externality to vary over the business cycle in the required manner, one needs the incentives for individual agents to choose less informative production techniques to increase in recessions. ${ }^{6}$ The source of changes in the strength of the externality will be the performance of the

\footnotetext{
${ }^{5}$ A similar type externality in the case of credit markets is examined in Lang and Nakumura (1990).

${ }^{6}$ A natural way of doing this would be to introduce risk aversion: the return on less socially informative investments are
} 
various production techniques as uncertainty about the unobserved factor fluctuates. When an agent is most uncertain about which of the two states the unobserved factor is in, the production technique that does not depend on the unobserved factor has the highest expected output. When an agent is certain about the state of the unobserved factor, production techniques that produce the most social information have the highest expected output. Production technique that do not depend on the unobserved factor produce the lowest expected output.

The level of certainty about the unobserved factor is interpreted as confidence. This provides a link to the Keynesian approach to economic fluctuations where fluctuations in the confidence of investors were a central driving force. In Keynesian theory increased uncertainty about appropriate investments leads to investors taking a stance of 'wait and see'. This was reflected by increased demand for liquid financial assets, particularly money. Here the investor stance of 'wait and see' is captured by production technique where no learning can take place about the underlying productivity factor.

Returning to the corridor analogy, the 'full coordination' path of the economy is when investor confidence is high. Under high confidence, the discrepancy between individual and social incentives is small. Displacements from the full coordination path will be caused by switches in the unobserved factor. The economy's reaction to these switches will determine the width of the corridor. If the reaction to the switches is a crisis of confidence then a spate of 'wait and see' type investments will be produced and confidence will remain at low levels with the economy outside the corridor. Alternatively, if the switches are understood quickly, high confidence will return and the economy will not leave the corridor.

Within the context of this corridor economy, one can attempt to simulate a depression by making the unobserved factor follow a very unlikely pattern. The pattern is designed to produce a big drop in confidence. The effects of the two types of government interventions described above are then considered. The externality means there is a system of subsidies to investment that will keep the economy on the full coordination path. Rather than considering such a sophisticated intervention, the simpler policy of

more certain because they do not depend on the unobserved factor. This is the mechanism used in Chalkley and Lee (1998). Using risk aversion adds a number of complications and also means that part of the asymmetries in business cycles will be dependent on the level of risk aversion. Since the level of risk aversion of individual agents is one of the most controversial issues in modern macroeconomics I prefer to restrict attention to risk neutral agents. 
subsidizing investments that produce information in recessions is used. The inept intervention consists of subsidizing the 'wait and see' type investment to mimic the actions of the Federal Reserve in contracting the money supply as described in Friedman and Schwartz.

The plan of the rest of the paper is as follows. Section 2 outlines the structure of the model in words. Section 3 characterizes the solution to the individual investment problem. Section 4 gives a precise description of the economic environment and gives a definition of confidence within the context of the model. Section 5 analyzes the dynamics of confidence with varying amounts of information available to individual investors. Section 6 presents simulation of a Great Depression type episode. Section 7 offers some brief conclusions. A short appendix describes some of the computations.

\section{The Model in Words}

The working of the model is best described by a simple parable. Each period a new generation of agents is born on a different island than previous generations. Agents live forever. The agents are endowed with two varieties of seeds to plant on the island in the first period of their life and a private signal about the productivity of the island. Any seeds that are not planted immediately perish. In the second period (i.e., one period after planting) the seeds produce two types of trees that have an infinite life and cannot be destroyed. The trees produce fruit subject to symmetric productivity shocks. There is exogenous technical progress over time so that the trees of later generations are on average more productive than the trees of earlier generations. However, the productivity of trees depreciates over time. The preferences of the representative individual in each generation are risk neutral and all types of fruit are perfect substitutes.

The marginal productivity of trees is determined by the type of soil on the island and the other trees planted. There are two types of soil. One variety of seeds produces more productive trees with soil

type 1. Another variety of seed produces more productive trees with soil type 2 . However, the soil of the island is such that if only one type of tree is used the land becomes infertile. Hence, a convex combination of the two types of seed is planted. If an equal amount of both varieties of seed is planted then the amount of fruit produced does not depend on the type of soil on the island. 
Not all the seeds can be planted because of space considerations on the island. The unconditional distribution of soil types across islands is uniform. Thus, on average, generations are born $1 / 2$ the time on soil type 1 islands and $1 / 2$ the time on soil type 2 islands. Moreover, if the current generation was born on a type 1 (2) island there is more than a $50 \%$ chance that the next generation will be born on a 1 (2) island.

The new generation is born knowing the unconditional distribution of soil over islands and the Markovian structure of soil types between adjacent generations and their islands. But they do not know the type of soil on their island. The optimal solution to their investment problem without further information is quite simple. Use a 50-50 split of seed types. This will be called the 'wait and see' investment. Alternatively, if the generation is born knowing the soil type on its island, then the convex combination of seeds planted will be at one of two extremes depending on the soil type. Before planting the seeds the new generation receives a private signal about soil type on its island.

Confidence is defined as the strength of belief about the soil type of the generation investing. Confidence is highest when they know the soil type and lowest when beliefs are equal to the unconditional distribution of soil types. In the cases of no information and full information, confidence is a degenerate random variable. Between these two extremes are the cases of

1. There is no trade or communication between islands. In this case the new generation has access only to its private signal. I will associate this information structure with the market structure of autarky. It will give the out of corridor behavior of the economy.

2. The new generation is assumed to have access to all the past investment decisions and individual outputs. This information structure will be associated with the market structure of a centralized stock market. That is, after making the investment, shares are issued for claims to fruit produced by individual islands. The dividends paid on these shares are equal to the output of the island.

Investment decisions produce information useful for future generations. Neither of the market structures reward investors for producing information. Hence even in the case of the stock market economy there will be some inefficiency. No attempt is made to solve the constrained first best solution. The analysis 
of the model consists in analyzing how different government interventions affect the stochastic properties of confidence through the amount of information available before planting the seeds. Two types of government intervention to correct inefficiencies are examined.

1. Subsidies: The government subsidizes an investment at one of the two extremes.

2. Inept: The government subsidizes the 'wait and see' investment decision.

Both types of intervention will be triggered by falls in aggregate output.

\section{The New Generations' Investment Decision}

The investors (new generation) born at time $s$ has one unit of uninstalled capacity (i.e., seeds) that must be split into two specific types of installed capacity (trees), $\left(x_{1 s}, x_{2 s}\right)$, in the following putty clay production function: ${ }^{7}$

$$
y_{t}\left(x_{1 s}, x_{2 s}\right)=A_{0}+A(s, t)\left(a+Z_{1 t} \ln \left(x_{1 s}\right)+Z_{2 t} \ln \left(x_{2 s}\right)\right)
$$

subject to:

$$
x_{1 s}+x_{2 s} \leq 1, x_{1 s} \geq 0, x_{2 s} \geq 0
$$

where $A_{0}$ is a positive constant that ensures that output is positive, $A(s, t)=\delta^{2 s-t}$ for $t \geq s, \delta>1$ $a=-\ln (0.5)$ and $Z_{1 t}, Z_{2 t}$ are independent and identically distributed random productivity shocks.

Although, $Z_{1 t}, Z_{2 t}$ are IID the new generation does not know their probability distribution function. Instead there is an unknown parameter $\lambda_{s}=0$ or 1 (soil type) that determines which of two possible combinations of random variables will generate the productivity shocks.

$$
\begin{aligned}
& Z_{1 t}=\lambda_{s} W_{1 t}+\left(1-\lambda_{s}\right) W_{2 t}, \\
& Z_{2 t}=\lambda_{s} W_{2 t}+\left(1-\lambda_{s}\right) W_{1 t},
\end{aligned}
$$

\footnotetext{
${ }^{7}$ The form of the production function is for computational and analytical convenience. In particular, it produces a simple form for the decision rule.
} 
where $W_{1 t}, W_{2 t}$ are independent of $\lambda_{s}$, IID over time and normally distributed. It is assumed that the parameters of the normal distributions are $E\left[W_{1 t}\right]=m_{1} \leq 1$ and $0 \leq E\left[W_{2 t}\right]=m_{2}$, with $m_{1}>m_{2}$ and $E\left[\left(W_{1 t}-m_{1}\right)^{2}\right]=E\left[\left(W_{2 t}-m_{2}\right)^{2}\right]=\sigma^{2}$.

Before making the investment the new generation receives a private signal, $\xi$ about $\lambda_{s}$.

$$
\xi=\lambda_{s} \zeta_{1}+\left(1-\lambda_{s}\right) \zeta_{2}
$$

where $\zeta_{1}, \zeta_{2}$ has a probability density function $f_{1}, f_{2}$ respectively, with $f_{1}(\zeta)=f_{2}(-\zeta)$. Investors combine this private signal with their initial beliefs about $\lambda_{s}$ using Bayes' Rule.

Let $\hat{b}(s, s)$ represent the belief of the new generation before observing their private signal. The first index represents the fact that these are beliefs about $\lambda_{s}$. The second index signifies the date of this belief. The hat is used to denote beliefs before receiving the private signal.

The belief after receiving the private signal is given by:

$$
b(s, s)=\frac{f_{1}(\xi) \hat{b}(s, s)}{f_{1}(\xi) \hat{b}(s, s)+f_{2}(\xi)(1-\hat{b}(s, s))} .
$$

The solution to the investment problem depends on predictions of the means of $Z_{1 t}, Z_{2 t}$. These predictions depend on beliefs, thus we use $\mathbf{E}_{b}$ to signify this.

The investment problem of the new generation with discount factor $\beta$ and assuming that the capacity has an infinite life is:

$$
\max _{x_{1 s}+x_{2 s}=1} \mathbf{E}_{b}\left[\sum_{t=s+1}^{\infty} \beta^{t-s} y_{t}\left(x_{1 s}, x_{2 s}\right)\right] .
$$

The first order conditions of the problem simplify to:

$$
\frac{\mathbf{E}_{b}\left[Z_{1 t}\right]}{x_{1 s}}=\frac{\mathbf{E}_{b}\left[Z_{2 t}\right]}{x_{2 s}}
$$

Setting $x_{s}=x_{1 s}$ and $x_{2 s}=1-x_{s}$ and normalizing so that $m_{1}+m_{2}=1$ gives the simple form for the decision rule:

$$
x_{s}=\mathbf{E}_{b}\left[Z_{1 t}\right] .
$$


This implies:

$$
x_{s}=\mathbf{E}_{b}[\lambda] E\left[W_{1 t}\right]+\mathbf{E}_{b}[(1-\lambda)] E\left[W_{2 t}\right]=m_{2}+b(s, s)\left(m_{1}-m_{2}\right) .
$$

Over time the generation will (passively) learn whether these initial beliefs were correct or incorrect. Using Bayes' rule again we have:

$$
b(s, t)=\frac{g_{1}\left(y_{t}\left(x_{s}\right)\right) b(s, t-1)}{g_{1}\left(y_{t}\left(x_{s}\right)\right) b(s, t-1)+g_{2}\left(x_{s}\right)(1-b(s, t-1))},
$$

where $g_{i}$ is the likelihood function for output given that $\lambda_{s}=i$ and $y_{t}\left(x_{s}\right)=y_{t}\left(x_{1 s}, x_{2 s}\right)$. Note that if $x_{s}=0.5$, the generation will not learn about the true value of $\lambda_{s}$ for their island since $g_{1}=g_{0}$.

In the case that $b(s, s)=1$, that is, $\lambda$ is known to equal 1 , output per period is normally distributed with mean:

$$
\mathbf{E}_{b(s, s)=1}\left[y_{t}\left(m_{1}\right)\right]=A_{0}+A(s, t)\left(a+m_{1} \ln \left(m_{1}\right)+m_{2} \ln \left(m_{2}\right)\right)=A_{0}+\delta^{2 s-t} \bar{y},
$$

and variance $\delta^{4 s-2 t} \bar{\sigma}^{2}$, where $\bar{\sigma}^{2}=\sigma^{2}\left(\ln \left(m_{1}\right)^{2}+\ln \left(m_{2}\right)^{2}\right)$. Since the productivity shocks and production function are symmetric this will also be the parameters of the normal distribution for output when $b(s, s)=$ 0 . In contrast, in the case that $b(s, s)=0.5$ the output per period is normally distributed with mean:

$$
\mathbf{E}_{b(s, s)=0.5}\left[y_{t}(0.5)\right]=A_{0}+A(s, t)(a+\ln (0.5))=A_{0},
$$

and variance $\delta^{4 s-2 t} \underline{\sigma}^{2}$, where $\underline{\sigma}^{2}=2 \sigma^{2} \ln (0.5)^{2}$.

\section{The Economic Environment}

The investment decisions of each generation have an obvious sequential ordering given by $s=1,2, \ldots$ It is convenient to index the islands in the same way. Each island is endowed with an embedded $\lambda_{s}$. Let $\lambda=\left\{\lambda_{s}: s=1,2, \ldots\right\}$ and $\Lambda$ is the set containing all possible $\lambda$.

Elements of $\Lambda$ are generated by a Markov Chain with initial probabilities for $\lambda_{0},(1 / 2,1 / 2)$ and symmetric transition probability $\alpha$. That is, with probability $\alpha$ the embedded value of productivity is the same across adjacent (in time) investment decisions and with probability $1-\alpha$ it switches. The 
simple Markov chain description of dynamics $\lambda$ maintains the symmetry of the individual decision at the aggregate level: Shocks are neither good nor bad. It depends on the interaction of shocks with past investment decisions.

The timing within a period is as follows: first all active islands produce; next, the private signal is revealed to the current generation; finally, the current generation makes its investment.

Fluctuations in the economy will be driven by beliefs. Let $\mathcal{B}$ represent the collection of probability measures over $\lambda$, with typical element B. Each joint distribution B contains marginal beliefs about the embedded productivity of each island or subsets of islands which we denote by, $b$, for example, $P\left[\lambda_{s}=\right.$ $1]=\sum_{\lambda_{t}: t \neq s} B\left[\ldots, \lambda_{s}=1, \ldots\right]$.

We need to distinguish between joint beliefs before and after the observation of a private signal. Let

$\hat{I}_{s}$ represent the information available to the generation investing before receipt of their private information. Thus, can focus on elements of $\mathcal{B}$ with the property:

$$
P\left[\lambda_{t}, t=1,2 \ldots, s \mid \hat{I}_{s}\right]=\hat{B}(s) .
$$

$I_{s}$ will be the information available to the current generation after observing their private signal. Define $B(s)$ to be the joint belief based on this (larger) information set.

It is convenient to take a simple function of beliefs and call it confidence. The most important belief concerns the new investment, $b(s, s)$. By construction $b(s, s)$ is between zero and one. Beliefs at either extreme represent highest confidence. Beliefs of $1 / 2$ represent the least confidence. Thus, confidence is defined by:

$$
c_{s}=2|b(s, s)-0.5|
$$

\section{Dynamics of Confidence and Aggregate Output}

In this section the dynamic behavior of confidence and aggregate output is described under varying amounts of information available to investors. 


\subsection{Full Information and No Information}

In the case of full information about $\lambda$ confidence is always equal to 1 and output of individual islands is drawn from a normal distribution as described above. Given the properties of $A(s, t)$ the mean and variance of the normal distribution for output decline over time for each island. Output across time for individual islands and across islands is independent. Hence, aggregate output is a Gaussian time series with an increasing mean:

$$
A_{0}(t-1)+\bar{y} \delta^{1-t} \frac{1-\delta^{2 t}}{1-\delta}
$$

and variance

$$
\bar{\sigma}^{2} \delta^{2-2 t} \frac{1-\delta^{4 t}}{1-\delta^{2}}
$$

In the case of no information confidence is always equal to zero and individual output for each island is normally distributed with mean $A_{0}$ and variance determined by $A(s, t)$. Once again individual outputs are independent across time and across islands. Thus, aggregate output is a Gaussian time series with increasing mean

$$
A_{0}(t-1)
$$

and increasing variance:

$$
\underline{\sigma}^{2} \delta^{2-2 t} \frac{1-\delta^{4 t}}{1-\delta^{2}}
$$

\section{$5.2 \quad$ Autarky}

Under autarky, only private information is available to the generation making the investment decision. The initial belief before observing the private signal can only be based on the probabilities of $\lambda_{0}$. Hence $\hat{b}(s, s)=0.5$. The updated belief depends on the private signal, $\xi$.

Since the private signal is IID across islands, confidence is symmetrically distributed around its mean value and is IID. Hence, given the symmetry of the rest of the model there can be no asymmetric business cycles and aggregate output will again be a Gaussian time series.

The accuracy of beliefs about the true $\lambda_{s}$ depend on the strength of the private signal. If the private signal is always very strong there will be little room for generating asymmetric business cycles by allowing 
observation of previous investment decisions and output. On the other hand, if there is little information in the private signal, the economy will remain in a state of low confidence forever, unless some information from previous investors can be transmitted to new investors. ${ }^{8}$

\subsection{Stock Market Economy}

Now, in addition to the private signal, assume that the generation making the investment can observe past investment decisions and the history of individual outputs. As discussed above this information structure is related to a market structure where the new generation makes its investment and then sells it on a centralized stock market. However, the construction of beliefs is first examined without using any pricing information from the stock market.

By sequentially updating, based on the previous investment decisions and the realization of individual outputs, the private signals can be obtained. First, note that using the investment rule the belief of the previous generations before making the investment decisions is:

$$
b(s, s)=\frac{x_{s}-m_{2}}{m_{1}-m_{2}} .
$$

In order to find the private signals received by the previous generation using, Equation 1, the generation investing needs to calculate the marginal belief held by the previous generation before observing the private signal. This requires that the new generation find the joint distribution $\hat{B}(s)$ and then extract from that the marginal belief $\hat{b}(s, s)$.

The updating starts with the investment of the first generation. They can generate $B(0)$ using the properties of the Markov chain. Note this produces uniform marginal distributions for each $s$. The belief $b(1,1)$ (revealed by the investment) is used to generate the joint belief $B(1)$ using the value of $\alpha$ and the Markov structure

\footnotetext{
${ }^{8}$ An alternative information structure that could also be supported by autarky would occur if only past investment decisions were observable and not output. In this case the model is similar to models of informational cascades. In models of informational cascades the actions of previous 'investors' can be sufficiently strong as to lead to current generation ignoring its private signal even when previous actions have been incorrect. However, there is a crucial difference produced by the Markovian structure. Even if the belief revealed by the previous generation's investment is 1 , or 0 the update by $\alpha$ leads to a belief for the present generation of $\alpha$ or $1-\alpha$.
} 
Next, using the recorded output from this initial investment, an update is made using Bayes' Rule:

$$
\hat{B}\left(\lambda_{1}=1, \lambda_{2}, \ldots\right) \propto g_{1}\left(y_{2}\left(x_{1}\right)\right) B\left(\lambda_{1}=1, \lambda_{2}, \ldots\right) .
$$

The marginal belief $\hat{b}(2,2)$ is obtained by averaging out over all values of $\lambda_{s}, s \neq 2$. Combining this with the belief $b(2,2)$ reflected in the investment $x_{2}$, the private signal $\xi_{2}$ received by the second generation can be obtained. This private signal is then used to find the joint belief $B(2)$ using Bayes' Rule again:

$$
B(2) \propto f_{1}\left(\xi_{2}\right) \hat{B}(2)
$$

Note that it is important to revise all of the beliefs at this time because the private signal on island 2 has information about $\lambda_{1}$.

The belief $B(2)$ is then updated using the record of individual outputs in period 3 to obtain $\hat{B}(3)$. This requires considering the likelihood function for 4 possible combinations of $\left\{\lambda_{1}, \lambda_{2}\right\}$ :

$$
g\left(y_{2}\left(x_{1}\right) \mid \lambda_{1}\right) g\left(y_{2}\left(x_{2}\right) \mid \lambda_{2}\right)
$$

The marginal belief $\hat{b}(3,3)$ is then combined with the investment decision of the third generation to find their private signal to produce $B(3)$. This process continues until the generation at time $s$ has found $B(s)$. They can then use this to find $b(s, s)$ using $\alpha$ and combine it with their private information to make their investment decision.

This process involves a great deal of computation. Further, much of it is wasteful since each generation would be repeating calculations made by previous ones. However, if one takes the stock market analogy seriously, then the number of calculations that each generation investing has to make is actually very small and also quite simple. The reason is that the stock market, through the operation of the 'Law of One Price', will construct marginal beliefs over claims to fruit on islands in production that are obtained from joint beliefs that reflect all available information.

Let $Q\left(x_{s}, b(s, t)\right)$ be the ex-dividend stock price of a claim to the future production of fruit on island $s$ at time $t$ and normalize the outstanding shares for the claim to fruit on each island to 1 . The expected 
present value (ex-dividend) of the output produced by the investment given belief $b(s, t)$ is:

$$
Q\left(x_{s}, b(s, t)\right)=\mathbf{E}_{b}\left[\sum_{j=1}^{\infty} \beta^{j} y_{t+j}\left(x_{s}\right)\right]
$$

using the definition of $y_{t}\left(x_{s}\right)$. After some algebra, the value can be split into a deterministic component, $Q_{1}\left(x_{s}\right)$ and a 'stochastic component' $b(s, t) Q_{2}\left(x_{s}\right)$, such that $Q\left(x_{s}, b(s, t)\right)=Q_{1}\left(x_{s}\right)+b(s, t) Q_{2}\left(x_{s}\right)$ with:

$$
\begin{gathered}
Q_{1}\left(x_{s}\right)=\frac{\beta}{1-\beta}\left(A_{0}+A(s)\left(a+m_{2} \ln \left(x_{s}\right)+m_{1} \ln \left(1-x_{s}\right)\right)\right), \\
Q_{2}\left(x_{s}\right)=\frac{\beta}{1-\beta}\left(A(s)\left(m_{1}-m_{2}\right)\left(\ln \left(x_{s}\right)-\ln \left(1-x_{s}\right)\right)\right) .
\end{gathered}
$$

$Q_{1}\left(x_{s}\right)$ will not change as observations on output are generated but $b(s, t) Q_{2}\left(x_{s}\right)$ will fluctuate since beliefs about the true value of $\lambda_{s}$ embedded with the investment will be updated given the observed output values from existing investments and investments made by new generations. ${ }^{9}$ Clearly it is possible to 'invert' stock prices for individual islands to find the market belief about the $\lambda$ value for this island.

In order to illustrate the restrictions imposed by the Law of One Price, consider the sum of the prices of two islands $s$ and $s^{\prime}$ respectively, with $s<s^{\prime}<t$ :

$$
Q\left(x_{s}, b(s, t)\right)+Q\left(x_{s^{\prime}}, b\left(s^{\prime}, t\right)\right)=Q_{1}(x)+Q_{1}\left(x^{\prime}\right)+b(s, t) Q_{2}(x)+b\left(s^{\prime}, t\right) Q_{2}\left(x^{\prime}\right)
$$

By the 'Law of One Price' this must trade for the same price as a claim to the output of both islands $s, s^{\prime}$. The value of this claim to the output on two islands depends on 4 possible events:

$$
\left\{\lambda_{s}=1, \lambda_{s^{\prime}}=1\right\},\left\{\lambda_{s}=1, \lambda_{s^{\prime}}=0\right\},\left\{\lambda_{s}=0, \lambda_{s^{\prime}}=1\right\},\left\{\lambda_{s}=0, \lambda_{s^{\prime}}=0\right\}
$$

The price of this claim can be evaluated under these 4 different events and then a belief weighted average of these values taken to obtain the price. This price depends on the joint beliefs about $\lambda_{s}, \lambda_{s^{\prime}}$ rather than the marginal beliefs contained in the prices of the individual firms:

$$
b(s, t)=P\left(\lambda_{s}=1, \lambda_{s^{\prime}}=1 \mid I_{t}\right)+P\left(\lambda_{s}=1, \lambda_{s^{\prime}}=0 \mid I_{t}\right),
$$

\footnotetext{
${ }^{9}$ Although agents in the model are risk neutral and productivity shocks are IID, the ex-dividend price will fluctuate until the true value of $\lambda_{s}$ is learnt.
} 


$$
b\left(s^{\prime}, t\right)=P\left(\lambda_{s}=1, \lambda_{s^{\prime}}=1 \mid I_{t}\right)+P\left(\lambda_{s}=0, \lambda_{s^{\prime}} \mid I_{t}\right) .
$$

These considerations must apply to all portfolios of active islands. Hence the collection of all marginal beliefs reflected in stock prices will be equivalent to the joint belief $\hat{B}(t)$ or $B(t)$.

The generation investing can look at the stock price of the most recent island after production at time $s$ to obtain $\hat{b}(s-1, s)$. It then updates using the Markov structure of $\lambda$ to find $\hat{b}(s, s)$. Next the private signal is received by the generation investing and they update to $b(s, s)$. Since their investment is publicly observable and $\hat{b}(s, s)$ is also known, once again the investment decision of the new generation can be used to find their private signal. Hence this information is included into the stock prices of active islands.

Consider the following two scenarios:

1. Most recent investments have been close to the 'wait and see' type.

Confidence will tend to be higher than the case of autarky because the information of the individual outputs is available and will have some additional power in identifying the $\lambda$ sequence. Thus, a weaker private signal will required to produce an informative investment. After such an investment, confidence will increase quickly whether or not the investment is successful, because the stock of information has increased. However, if private signals are sufficiently weak and confidence is low for an extended period of time the economy might loose all of its stock of knowledge on $\lambda$ and for some time behave in a similar manner to the case of autarky. This would represent out of corridor behavior.

2. Most previous investments have been close to the 'extreme' type and the unobserved factor switches. Aggregate output declines will be more severe than in the case of autarky because the higher level of confidence will have lead to more extreme investments and hence bigger mistakes. However, the source of the output decline should be easy to determine even if some recent investments are close to the 'wait and see' type. This is because unexpectedly low output is very informative about the path of $\lambda$ in the recent past. Also if there is sufficient persistence in the Markov states this information 
will be useful for current investment. Thus, confidence is likely to return quickly. In particular, once confidence declines it can return to high levels either through good or bad news.

The stock market economy could still leave the corridor as outlined at the end of scenario 1 . Alternatively, if the government followed the perverse policy of subsidizing the 'wait and see' investment in recessions, the self-stabilizing property of the stock market economy would be greatly weakened. Note that, besides reducing the information content of individual outputs, it would also make it impossible to recover the private signals thus a state worse than autarky could occur.

In the case of the subsidizing extreme investments, the information content of individual outputs is maximized but the private signal is still not communicated to other agents. Further, by definition half of the time when confidence is low extreme investments will be exactly wrong. This could have a large negative effect on output because the subsidies will be activated when output drops, but this does not necessarily imply that confidence is low.

\section{Simulating A Depression}

The objective is to analyze differences in the propagation of adverse shocks produced for the stock market economy with the two types of government intervention. To accomplish this task a particular path is imposed on the $\lambda$ sequence. The frequency of observation is taken to be monthly. For 120 periods $\lambda_{t}$ is fixed at the value of 1 and transitions to 0 are prevented. This means that confidence will build up to high levels in the stock market economy. Then a sequence of alternating values of $\lambda_{t}$ is imposed for 12 periods. The aim is to lower confidence as much as possible. After these restrictions the movement of $\lambda$ is returned to the control of the Markov chain.

The economy is simulated under the same set of individual productivity shocks and private signals for each government intervention. Hence, any differences in the time path of aggregate output will be attributable to the different propagation mechanisms produced by the government interventions. To isolate the effect of the sequencing of $\lambda_{t}$, the simulations are repeated 50 times with the $\lambda$ sequence fixed but with

the individual productivity shocks and private signals changing. These simulations are then averaged to 
obtain the behavior of the economy under the adverse $\lambda$ sequence.

Various parameters have to be chosen before the model is simulated. Perhaps the most important are $\alpha$ and the private signal. For example, if $\alpha=0.5$ then the behavior of the stock market economy and autarky will be identical. Alternatively, if $\alpha=1$, very quickly the individuals in the stock market economy will know the whole $\lambda$ sequence with certainty. Hence, $\alpha$ is set to 0.95 .

As discussed above, if private signals are highly informative and $\alpha<1$, there will be little difference between autarky and the stock market economy. The private signal is assumed to be generated by a normal distribution, with mean of 1 if $\lambda_{s}=1$ and a mean of -1 if $\lambda_{s}=0$, and a standard deviation of 10 implying private signals are very weak.

For the parameters of the normal distribution of the productivity shocks, $m_{1}$ is set to 0.67 and $m_{2}$ to 0.33. The standard deviation is set to 0.1 . Given $b(s, s)=1$, the difference in output means between $\lambda_{s}=1$ and $\lambda_{s}=0$ at the optimal investment is approximately 0.24 with a standard deviation of approximately 0.12. Thus, individual output values will produce very quick learning about the true $\lambda$. Alternatively, if $b(s, s)$ is 0.75 the difference in ouput means is approximately 0.12 at the optimal investment with a standard deviation of approximately 0.1 and the true value of $\lambda$ will not be learnt as quickly.

The final parameter to choose is the exogenous rate of technological progress. The rate is chosen by considering the case where the $\lambda$ sequence is observable. This represents the maximum growth rate and it is fixed at approximately a $6 \%$ annual rate. This implies that $\delta \approx 0.007$ per month. ${ }^{10}$

The government subsidy intervention is defined to occur if aggregate output falls over a period of six months, the usual definition of a recession. The intervention is restricted to start only after the alternating sequence of $\lambda_{t}$ has occurred and consists of a subsidy for an extreme investment only while the economy is in recession. The inept government intervention starts after the alternating sequence of $\lambda_{t}$ and lasts for 24 periods.

The results of the simulation are presented graphically. First consider the behavior of confidence under the three cases. Figures 3, 4 and 5 show the dynamics of confidence for the cases of no intervention,

\footnotetext{
${ }^{10}$ This calculation ignores $A_{0}$ and transition effects in $A(s, t)$.
} 


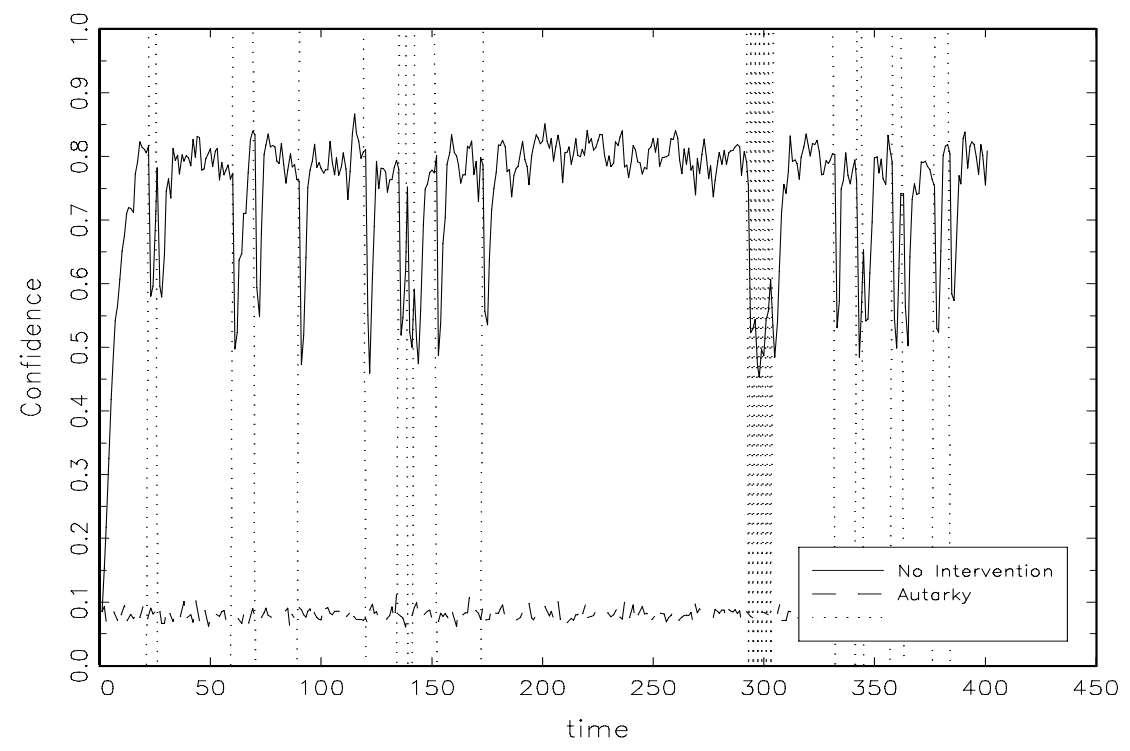

Figure 3: Confidence in the Stock Market Economy with no Government Intervention

investment subsidies and inept intervention respectively. Each graph also contains the level of confidence in the case of autarky. The vertical lines in each graph represent the occurrences of switches in the underlying $\lambda$ sequence. Note that the dynamics of confidence in all three graphs are the same until just after observation 300 when the different policies start.

There appears to be little difference in the dynamics of confidence in the cases of no government intervention and investment subsidies with both exhibiting a strong bounce back in confidence after the alternating sequence of $\lambda$ s. Moreover, the drop in confidence from the engineered alternating sequence does not seem to be any deeper from that produced by early or later random switches in $\lambda$ except for its duration. On the other hand, the inept government intervention drives confidence down to that found in the autarkic economy. However, once the inept policy is 'turned-off' the economy returns quickly to the previous level of confidence. Examination of the output sequences shows that the bounce back in 


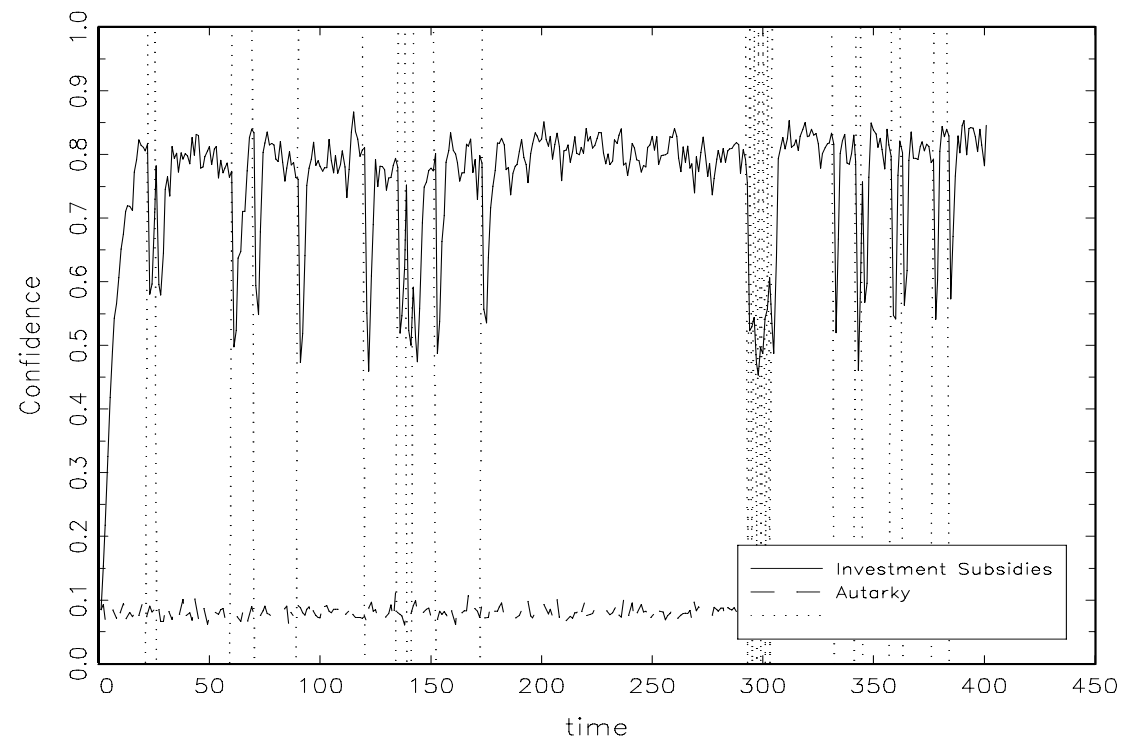

Figure 4: Confidence in the Stock Market Economy with Investment Subsidies

confidence observed after switches occurs for both good and bad news.

Figure 6 contains the path of the natural logarithm of aggregate output in the three cases and the full information level. Policy differences only occur when the alternating sequence has finished. The common effect of the adverse information shock is a drop in aggregate output of almost $20 \%$. The drop is not persistent in the case of no intervention or investment subsidies, with output returning to its predepression level 36 periods after the alternating sequence. However, this still represents a considerable loss of efficiency relative to the full information case compared to the pre-depression period (that is, the ratio drops from 0.8 to 0.7 .) For the inept intervention the economy continues to decline, because there is no new investment other than the 'wait and see' type, and the decline in output is $25 \%$ for a considerable time.

Finally, Figure 7 shows the behavior of the value of stock market across the three scenarios. No- 


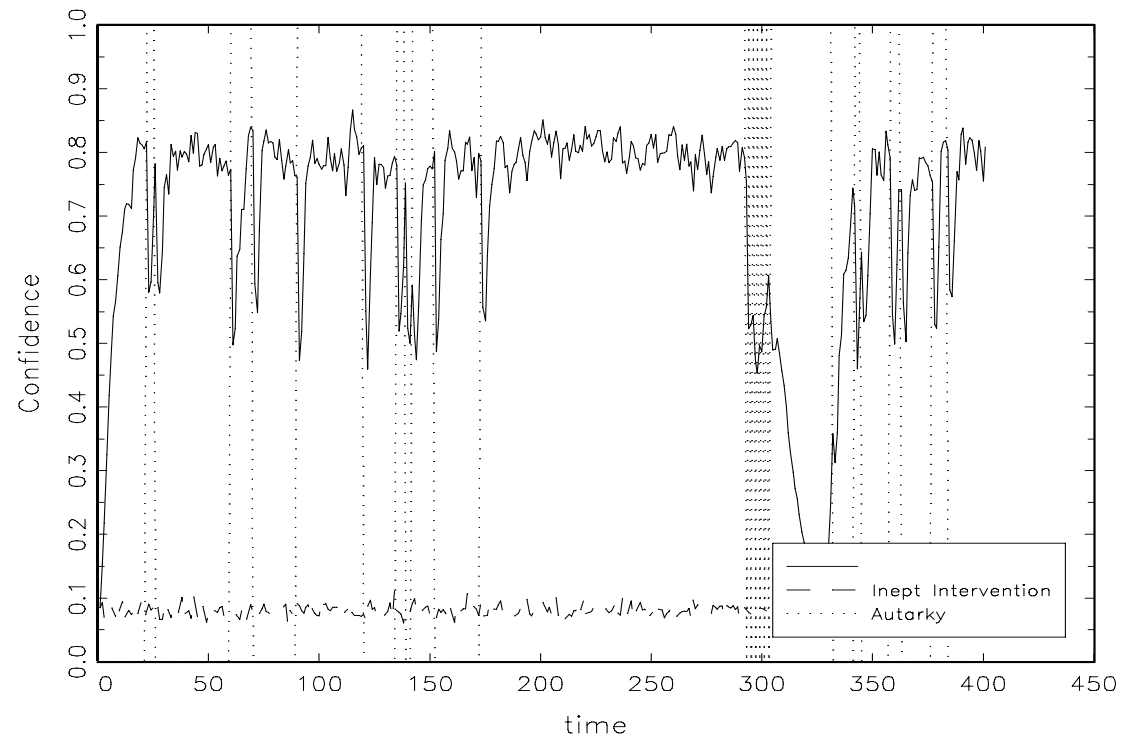

Figure 5: Confidence in the Stock Market Economy with Inept Intervention

tice how the alternating pattern of $\lambda$ produces a large drop in stock market value. Under the cases of no intervention and investment subsidies, this drop is reversed quickly but it continues under the inept intervention throughout the 50 periods shown with a cumulative drop of $22 \%$. 


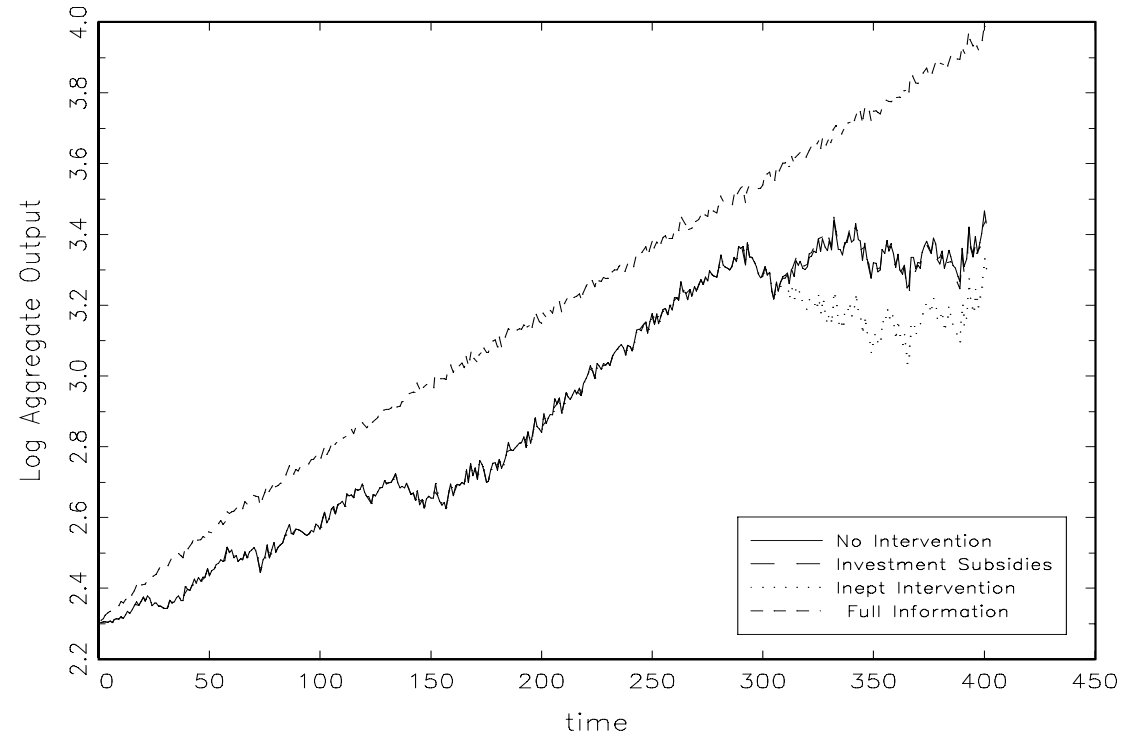

Figure 6: Aggregate Output under various Interventions 


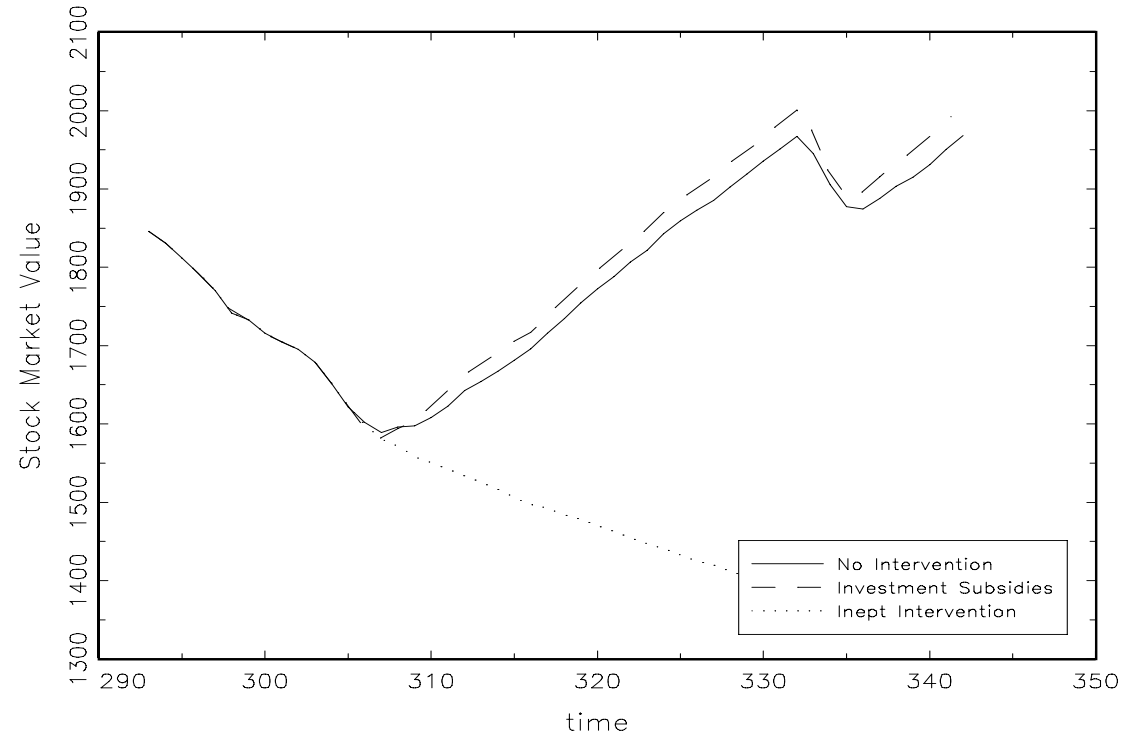

Figure 7: Stock Market Values during the Depression period 


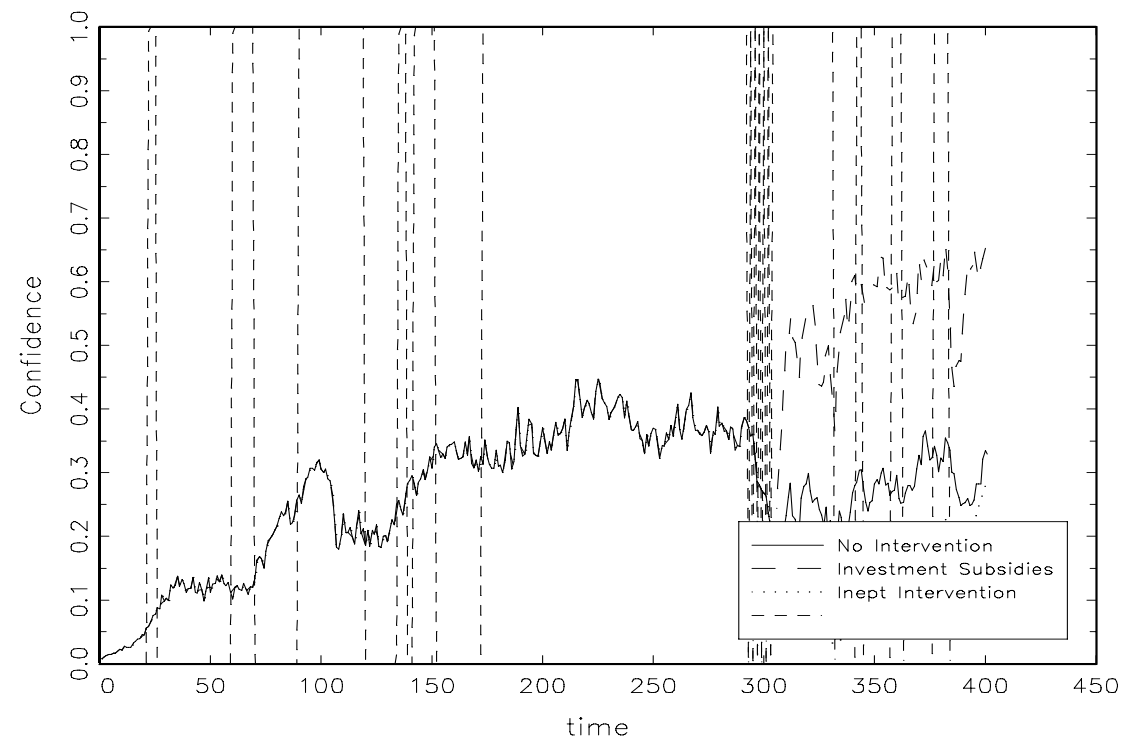

Figure 8: Confidence with and without investment subsidies in a noisier economy

The chosen parameter values for the model do not allow investment subsidies much of a role in raising investor confidence. However, if we decrease the informativeness of the private signal and make the signal from individual outputs weaker, then investment subsidies can lead to a big increase in confidence. This is illustrated in Figure 8 for the case where the standard deviation of the private signal is 100 and the standard deviation of $W_{i t}$ is 0.3 . Notice how confidence is very low until the investment subsidies start and then increases relative to the no intervention case. However, the implied dynamics for aggregate output are counterfactual: Output growth before the induced depression is negligible.

\section{Conclusions}

There is now a great deal of empirical evidence that business cycle fluctuations contain asymmetries. Specifically, post-war US output appears to be resistant to negative shocks during recessions. This asym- 
metry is inconsistent with the Great Depression period. One natural explanation for this inconsistency is changes in government policy. A theoretical explanation for asymmetries based on rational fluctuations in the confidence of investors is developed. It is used to address the role that government intervention might have played in producing this inconsistency. It is found that the 'ineptness' of government intervention during the Great Depression in reducing the confidence of investors rather than the success of post-war stabilization policy in raising confidence is the most likely explanation. 


\section{Appendix}

In this appendix the algorithm of Kim (1994) is applied to the updating problem of the model. In the description of section 5 beliefs were updated using the joint distribution $B(t)$. Such updating quickly becomes infeasible since the order of $2^{t}$ different sequences have to be followed. Instead the simulations of section 6 are calculated in a recursive manner. For each island $s$ define $\ell_{i}(s, t)$ to be the likelihood that $\lambda_{s}=i$ given information up to time $t$ produced on island $s$, that is the history of output and the private signal. Then starting from $b(1,0)=0.5$ we have using Bayes Rule:

$$
\tilde{b}(1, t, 1)=\frac{\ell_{1}(1, t)}{\ell_{1}(1, t)+\ell_{2}(1, t)}
$$

Where the first argument of $b$ represents the island, the second argument the current period and the third argument the number of islands whose information is included in the belief. Using the partial update from the first island we have:

$$
\tilde{b}(2, t, 1)=\alpha \tilde{b}(1, t, 1)+(1-\alpha)(1-\tilde{b}(1, t, 1))
$$

Now using Bayes Rule we have

$$
\tilde{b}(2, t, 2)=\frac{\tilde{b}(2, t, 1) \ell_{1}(2, t)}{\tilde{b}(2, t, 1) \ell_{1}(2, t)+(1-\tilde{b}(2, t, 1)) \ell_{2}(2, t)} .
$$

This process continues until $\tilde{b}(t, t, t)$ is obtained. Here the information from island $t$ is its private signal. For simulating aggregate output this forward recursion would be adequate. However, to find stock market values we need to smooth the beliefs by introducing the same information to each island. Following Kim (1994) this can be achieved by the backwards recursion:

$$
b(s, t, t)=b(s, s, s)\left[\alpha \frac{b(s+1, t, t)}{b(s+1, s, s)}+(1-\alpha) \frac{(1-b(s+1, t, t))}{(1-b(s+1, s, s))}\right]
$$




\section{References}

[1] Balke, N., and Wynne, M. (1994), "The dynamics of recoveries," Federal Reserve Bank of Dallas working paper 94-06.

[2] Beaudry, P. and G. Koop (1993). "Do recessions permanently change output?" Journal of Monetary Economics, 31, 149-164.

[3] Bikhcandani, S., Hirshliefer, D., and Welch, I., (1992), "A theory of fads, fashion, custom and cultural change as information cascades," Journal of Political Economy, 100, 992-1026.

[4] Chalkley, M., and Lee, I.H. (1998) "Asymmetric business cycles" Review of Economic Dynamics forthcoming.

[5] Cooper, R., and John, A., (1988), Coordinating coordination failures in Keynesian models," Quarterly Journal of Economics 103, August, 441-463.

[6] Friedman, M., (1993) "The plucking model of economic fluctuations revisited," Economic Inquiry 31, April, 171-177.

[7] Friedman, M., and Schwartz, A. (1963) A Monetary History of the United States 1857-1960, NBER, Chicago.

[8] Hamilton, J.D. (1989). "A new approach to the economic analysis of nonstationary time series and the business cycle," Econometrica, 57, 357-384

[9] Kim, C-J. (1994). "Dynamic Linear Models with Markov-Switching." Journal of Econometrics,

[10] Lang, W. and Nakamura, L., (1990). "The dynamics of credit markets in a model with learning," Journal of Monetary Economics 26, 305-318.

[11] Leijonhufvud, A., (1981), Information and Coordination, Oxford University Press, Oxford.

[12] Neftci, S. (1984), "Are economic time series asymmetric over the business cycle?" Journal of Political Economy, 92, 307-328.

[13] Potter, S.M., (1995a). "A nonlinear approach to US GNP," Journal of Applied Econometrics, 10, 109-126.

[14] Tiao, G., and Tsay, R., (1994): "Some Advances in Nonlinear and Adaptive Modeling in Time Series Analysis," Journal of Forecasting, 13, 109-131. 